\title{
State-dependent signatures of Anti-NMDA-Receptor Encephalitis: a dynamic functional connectivity study
}

\author{
Nina von Schwanenflug ${ }^{1,2}$, Stephan Krohn ${ }^{1,2}$, Josephine Heine ${ }^{1}$, Friedemann Paul ${ }^{1,3,4,5}$ Harald \\ Prüss $^{1,6}$, Carsten Finke ${ }^{1,2}$ \\ ${ }^{1}$ Department of Neurology, Charité - Universitätsmedizin Berlin, Corporate Member of Freie \\ Universität Berlin, Humboldt-Universität zu Berlin, and Berlin Institute of Health, Berlin, Germany \\ ${ }^{2}$ Berlin School of Mind and Brain, Humboldt-Universität zu Berlin, Berlin, Germany \\ ${ }^{3}$ Experimental and Clinical Research Center, Max Delbrueck Center for Molecular Medicine and \\ Charité - Universitätsmedizin Berlin, Berlin, Germany \\ ${ }^{4}$ Berlin Institute of Health, Berlin, Germany \\ ${ }^{5}$ NeuroCure Clinical Research Center, Charité - Universitätsmedizin Corporate Member of Freie \\ Universität Berlin, Humboldt-Universität zu Berlin, and Berlin Institute of Health, Berlin, Germany \\ ${ }^{6}$ German Centre for Neurodegenerative Diseases, DZNE, Berlin, Germany
}

Keywords: autoimmune encephalitis, functional connectivity, dynamic functional connectivity, machine learning 


\section{ABSTRACT}

\section{Introduction}

Anti-N-methyl-d-aspartate receptor encephalitis (NMDARE) is an autoimmune disorder associated with severe neuropsychiatric symptoms. While patients with NMDARE exhibit disrupted functional connectivity (FC), these findings have been limited to static connectivity analyses. This study applies time-resolved FC analysis to explore the temporal variability of large-scale brain activity in NMDARE and to assess the discriminatory power of functional brain states in a supervised classification approach.

\section{Methods}

Resting-state fMRI data from 57 patients with NMDARE and 61 controls was included. To capture brain dynamics, four discrete connectivity states were extracted and state-wise group differences in FC, occurrence, dwell time and transition frequency were assessed. Furthermore, logistic regression models with embedded feature selection were trained for each state to predict group status in a leave-one-out cross validation scheme.

\section{Results}

Patients showed FC alterations in three out of four states. Besides a reduction in hippocampalfrontal connectivity, we observed connectivity decreases within the default mode network and between frontal areas and subcortical as well as visual regions, which remained undetected in static FC. Furthermore, patients displayed a shift in dwell time from the weakly connected dominant state to a higher connected, but less frequent state, accompanied by increased transition frequencies. Discriminatory network features and predictive power varied dynamically over states, reaching up to $78.6 \%$ classification accuracy.

\section{Conclusion}

Patients showed state-specific alterations in FC along with a shift in dwell time and increased volatility of state transitions. These measures were associated with disease severity and duration, highlighting the potential of spatiotemporal dynamics in FC as prognostic biomarkers in NMDARE. 


\section{INTRODUCTION}

Anti-N-methyl-d-aspartate receptor encephalitis (NMDARE) is a severe autoimmune disorder of the central nervous system that is caused by antibodies targeting the NR1 subunit of the NMDA receptor (1). NMDARE is characterized by a neuropsychiatric syndrome including movement disorders, psychosis, autonomic dysfunction, decreased levels of consciousness and cognitive dysfunction, e.g. deficits of executive control and memory (1-6). Despite the severe disease course, routine clinical magnetic resonance imaging (MRI) reveals no abnormalities in 50 to $80 \%$ of patients $(6,7)$. In contrast, functional connectivity (FC) is disrupted in several resting-state networks, with a focus on sensorimotor, fronto-parietal, lateral-temporal, and visual networks (8). Furthermore, hippocampal connectivity to medial frontal regions of the default-mode network is significantly reduced in NMDARE, and these alterations are associated with the severity of memory impairment $(3,8)$.

Resting-state FC analyses have thus contributed to reveal some of the mechanisms underlying clinical symptoms in NMDARE. These now well-established analyses characterize the functional architecture of the brain by assessing the coherence of brain activity between distinct regions.

However, traditional FC analyses are 'static' in the sense that blood-oxygen-level dependent (BOLD) time series are averaged across a several minute scan (9). In fact, the brain is a complex dynamic system in which strength and spatial organization of connectivity patterns change at a much faster timescale (seconds apart) resulting in multiple spatiotemporal organisations within one scan (10-12). Hence, static FC omits the opportunity to capture dynamic changes of functional brain organization and to obtain potential information that can be employed in the characterization of psychiatric or neurological diseases $(10,11,13,14)$. Accordingly, a growing number of studies found intriguing evidence that FC fluctuations are related to the subject's internal state (15-17) and add valuable information to the understanding of disease development and progression $(13,18-21)$. One common method to analyze time-varying FC applies a clustering algorithm to obtain distinct functional brain states, which are defined as quasi-stable spatiotemporal patterns of FC that reoccur during a restingstate scan (22).

Following this methodology, the present study aimed to characterize dynamic signatures of brain activity in NMDARE and to investigate the association of spatiotemporal FC patterns to disease duration and severity in a large patient cohort. We hypothesized that the temporal dynamics of FC would be altered in NMDARE patients compared to controls. Furthermore, we expected unique connectivity changes for each state in the group comparisons, emphasizing a difference in relevance of states for the characterization of the disease. Additionally, we hypothesized that the predictive potential of distinct dynamic FC states to discriminate between 3 
bioRxiv preprint doi: https://doi.org/10.1101/2020.06.12.141945; this version posted June 12, 2020. The copyright holder for this preprint (which was not certified by peer review) is the author/funder. All rights reserved. No reuse allowed without permission.

patients with NMDARE and healthy controls $(\mathrm{HC})$ would vary over brain states, which we investigated in a supervised binary classification approach. 


\section{METHODS}

\section{Participants}

In this study, 57 patients with NMDARE (50 females; median age: 25 years) were recruited from the Department of Neurology at Charité-Universitätsmedizin Berlin. Diagnosis was based on clinical presentation and detection of IgG NMDAR antibodies in the cerebrospinal fluid. Disease severity at the time of scan was assessed with the modified Rankin scale (mRS). The control group consisted of 61 age- and sex-matched healthy participants (54 females; median age of 26 years; $\mathrm{t}(116)=0.724 ; \mathrm{p}=0.342$ ) with no history of neurological and/or psychiatric disease. Clinical and demographic characteristics are summarized in Table 1. All participants gave written informed consent and the study was approved by the local ethics committee.

\section{MRI data acquisition}

Structural and functional MRI data were acquired at the Berlin Center for Advanced Neuroimaging at Charité-Universitätsmedizin Berlin using a 20-channel head coil and a 3T Trim Trio scanner (Siemens, Erlangen, Germany). For resting-state functional MRI (rs-fMRI), we employed an echoplanar imaging sequence $(\mathrm{EPI} ; \mathrm{TR}=2.25 \mathrm{~s}, \mathrm{TE}=30 \mathrm{~ms}, 260$ volumes, matrix size $=64 \times 64,37$ axial slices aligned to the A-P plane, slice thickness $=3.4$, voxel size $=3.4 \times 3.4 \times 3.4 \mathrm{~mm}^{3}$ ). Participants were instructed to lie still, close their eyes and stay awake. High-resolution T1-weighted structural scans $\left(1 \times 1 \times 1 \mathrm{~mm}^{3}\right)$ were collected using a magnetization-prepared rapid gradient echo sequence (MPRAGE; matrix size $=240 \times 240$, 176 slices).

\section{MRI data analysis}

Our processing pipeline followed the procedure of recent related work $(13,22)$. Preprocessing of rs-fMRI scans included discarding the first 5 volumes to account for equilibration effects, slice time correction, realignment to the first volume, spatial normalization to MNI space (voxel size $2 \times 2 \times 2 \mathrm{~mm}$ ), and spatial smoothing with a $6 \mathrm{~mm}$ full width at half maximum smoothing kernel using the CONN Toolbox (https://web.conn-toolbox.org/).

\section{Group independent component analysis}

To perform group-independent component analysis (ICA) and dynamic functional network analysis, we applied the GrouplCA fMRI toolbox (GIFT, http://mialab.mrn.org/software/gift/index.html). For each participant, 255 timepoints were first 
decomposed into 150 temporally independent principle components (PC) and subsequently into 100 independent PCs using the Infomax algorithm (23). This procedure was repeated 20 times in ICASSO (24) to estimate reliability and ensure stability of the decomposition. For backreconstruction of individual timecourses and spatial maps, gig-ica (integrated in the GIFT Toolbox) was applied to the data (25). The resulting 100 independent components were individually rated as signal or noise by two independent raters based on functional network labels proposed by Yeo and colleagues (26). In total, 39 components were assigned to functional networks based on the labels proposed by Yeo and colleagues (26). For cerebellar and subcortical components, two distinct networks were added. This yielded a total of seven functional resting-state networks including sensorimotor (SM), visual (VIS), subcortical (SB), cerebellar (CB), default mode (DMN), dorsal attention (dATT), and frontoparietal control network (FPN). Supplementary Figure 1 shows all functional networks and Supplementary Table 2 contains peak values and coordinates for all components. Finally, we applied additional processing steps including linear, quadratic, and cubic detrending, motion regression (12 motion parameters) to reduce motion-related artifacts, high-pass filtering $(0.15 \mathrm{~Hz}$ cut-off), despiking (identified as framewise displacement $>0.5 \mathrm{~mm}$ ) and interpolation of timecourses using a 3rd order spline fit.

\section{Static functional network connectivity analysis}

To compare the dynamic FC results with conventional 'static' FC, we first calculated the average connectivity between all component pairs over the whole timecourse. We extracted each participant's preprocessed component time series and calculated Spearman's $\rho$ between every component pair. Subsequently, age, sex and motion parameters were regressed out and Fisher-Z transformation was applied. Then, all correlation matrices were averaged for each group separately for visualization.

\section{Dynamic functional network connectivity analysis}

In order to obtain FC dynamics, FC between all component pairs was calculated over consecutive windowed segments of the timecourses (i.e., sliding windows) (27) using a window of 30TR length ( $\triangleq 67.5$ seconds) that shifted in steps of $1 \mathrm{TR}(\hat{=} 2.25$ seconds). Rectangular windows were convolved with a Gaussian of $\sigma=3$ TRs to obtain tapering along the edges. After the correlation matrix was computed on each window (i.e., $22539 \times 39$ matrices per participant), Fisher-Z transformation was applied and age, sex and motion parameters were regressed out as nuisance variables. Subsequently, matrices of each participant were concatenated (26550 matrices in total) and k-means clustering was applied with k = 4 
according to the elbow criterion (see Supplementary Figure 2). Thus, each window was assigned to one of 4 clusters representing discrete network FC states (22). Squared Euclidean distance was applied for clustering, and the process was repeated 100 times to avoid convergence on local minima.

\section{Group differences in static and dynamic functional network connectivity (FNC)}

Next, we assessed group differences in FC for the static and the dynamic approach with respect to connectivity strength. For the latter, group differences were assessed for each state separately. Static as well as state-wise between-group comparisons were considered a family of univariate tests, where adjustment for multiple comparisons according to Benjamini \& Hochberg (28) was applied to control the false discovery rate (FDR).

Besides the analysis of state-dependent connectivity patterns, estimation of time-varying FC provides the opportunity to capture dynamic metrics. Here, three commonly used metrics were calculated: (a) state occurrence rate (i.e., number of participants that entered the state over the course of the scan), (b) dwell time (i.e., total number of windows a participant spends in a particular state) and (c) transition frequency (i.e., a participants' total number of transitions between each pair of states) $(13,29)$.

Between-group comparisons for the static and dynamic functional network analysis as well as dwell time and transition frequencies were based only on participants that visited the respective state and were calculated using a "non-parametric t-test", which uses permutations to estimate the null distribution (30). Group differences in occurrence rates were calculated using the ztest for population proportions.

\section{State-wise classification}

Finally, group-wise analyses were complemented with a supervised binary classification approach to assess the potential of the static FC markers and the four dynamic FC states to discriminate between patients and controls. As previous work has suggested visual, frontoparietal, and default mode network (DMN) areas to represent the biologically relevant discriminatory features in NMDARE (8), these networks were considered as the set of input features. For the static design and for each state, logistic regression models were trained on the z-scored FC indices to predict group status (NMDARE patients vs. HC) in a leave-one-out cross validation (LOOCV) scheme. To facilitate model sparsity and counteract overfitting, embedded feature selection was applied through L1 regularization. Hyperparameter optimization of the regularization strength $\lambda$ was applied for each state input matrix 
(observations-by-connectivity features) by searching a linearly spaced parameter grid that was identical for all four states. Selection probability of each feature was read out as the empirical rate of non-zeroed feature weights over all predictions within a state. Prediction performance was evaluated by standard confusion matrix measures (i.e., true and false positive and negative rates and overall accuracy). Model training and prediction was implemented with the LIBSVM package for Matlab (The MathWorks, Inc., Natick, MA). 


\section{RESULTS}

\section{Functional Network Analysis}

\section{Static functional network connectivity analysis}

Differences in static FC between NMDARE patients and HC clustered in the inter- and intraconnectivity of the DMN and the FPN (Figure 1 and Table 2). NMDARE patients showed significantly decreased static connectivity between the hippocampus and the medial prefrontal cortex (mPFC; $\left.p_{F D R}<0.05\right)$. Apart from the hippocampus, frontal areas of the DMN were primarily affected. While patients with NMDARE displayed decreased connectivity for most of the component pairs, connectivity between the mPFC and the bilateral orbitofrontal gyrus was increased compared to controls ( puncorr $_{2} 0.001$ ). Additionally, connectivity between visual network and dorsal attention network regions was decreased in NMDARE patients compared to $\mathrm{HC}$ (TPO-junction and the parieto-occipital sulcus to frontal areas).

Table 1: Demographic variables and clinical measures of the participants. Table lists median and interquartilerange $(\mathrm{IQR})$ of respective variables. Disease duration = days in hospitalization; $\mathrm{N}=$ number of subjects; mRS = modified Rankin Scale.

\begin{tabular}{|c|c|c|c|}
\hline & & NMDARE Patients & Healthy Controls \\
\hline $\mathbf{N}$ & & 57 & 61 \\
\hline Sex & +10 & $50 / 7$ & $54 / 7$ \\
\hline Age (years) & $\begin{array}{c}\text { Median } \pm I Q R \\
(N)\end{array}$ & $25.00 \pm 14.50(56)$ & $26.00 \pm 11.00(61)$ \\
\hline Maximum mRS & $\begin{array}{c}\text { Median } \pm \text { IQR } \\
(\mathrm{N})\end{array}$ & $4.00 \pm 2.00(50)$ & $\mathrm{n} / \mathrm{a}$ \\
\hline mRS at scan & $\begin{array}{c}\text { Median } \pm \text { IQR } \\
(\mathrm{N})\end{array}$ & $1.00 \pm 1.00(50)$ & $\mathrm{n} / \mathrm{a}$ \\
\hline Disease duration & $\begin{array}{l}\text { Median } \pm \text { IQR } \\
(\mathrm{N})\end{array}$ & $63.00 \pm 56.50(45)$ & $\mathrm{n} / \mathrm{a}$ \\
\hline $\begin{array}{c}\text { N subjects with first-line } \\
\text { treatment }\end{array}$ & yes/no/unknown & $51 / 1 / 5$ & $\mathrm{n} / \mathrm{a}$ \\
\hline $\begin{array}{c}\text { N subjects with second- } \\
\text { line treatment }\end{array}$ & yes/no/unknown & $27 / 24 / 6$ & $\mathrm{n} / \mathrm{a}$ \\
\hline $\begin{array}{l}\text { N subjects with } \\
\text { anticonvulsants }\end{array}$ & yes/no/unknown & $39 / 12 / 6$ & $\mathrm{n} / \mathrm{a}$ \\
\hline $\begin{array}{l}\mathrm{N} \text { subjects with } \\
\text { antipsychotics }\end{array}$ & yes/no/unknown & $37 / 15 / 5$ & $\mathrm{n} / \mathrm{a}$ \\
\hline
\end{tabular}


bioRxiv preprint doi: https://doi.org/10.1101/2020.06.12.141945; this version posted June 12, 2020. The copyright holder for this preprint (which was not certified by peer review) is the author/funder. All rights reserved. No reuse allowed without permission.

Following previous studies that found a correlation between the mPFC-hippocampal connection and disease severity variables $(3,8)$, we conducted a post-hoc correlation analysis (using Pearson's correlation coefficient) between these regions and disease severity at the time of scan (mRS). Higher mRS was significantly associated with a reduced connectivity between the parahippocampal gyrus and the mPFC $(R=-0.33, p=0.029)$, as well as a lower connection between the hippocampus and the $\operatorname{mPFC}(R=-0.25, p=0.081)$, albeit limited to statistical tendency.
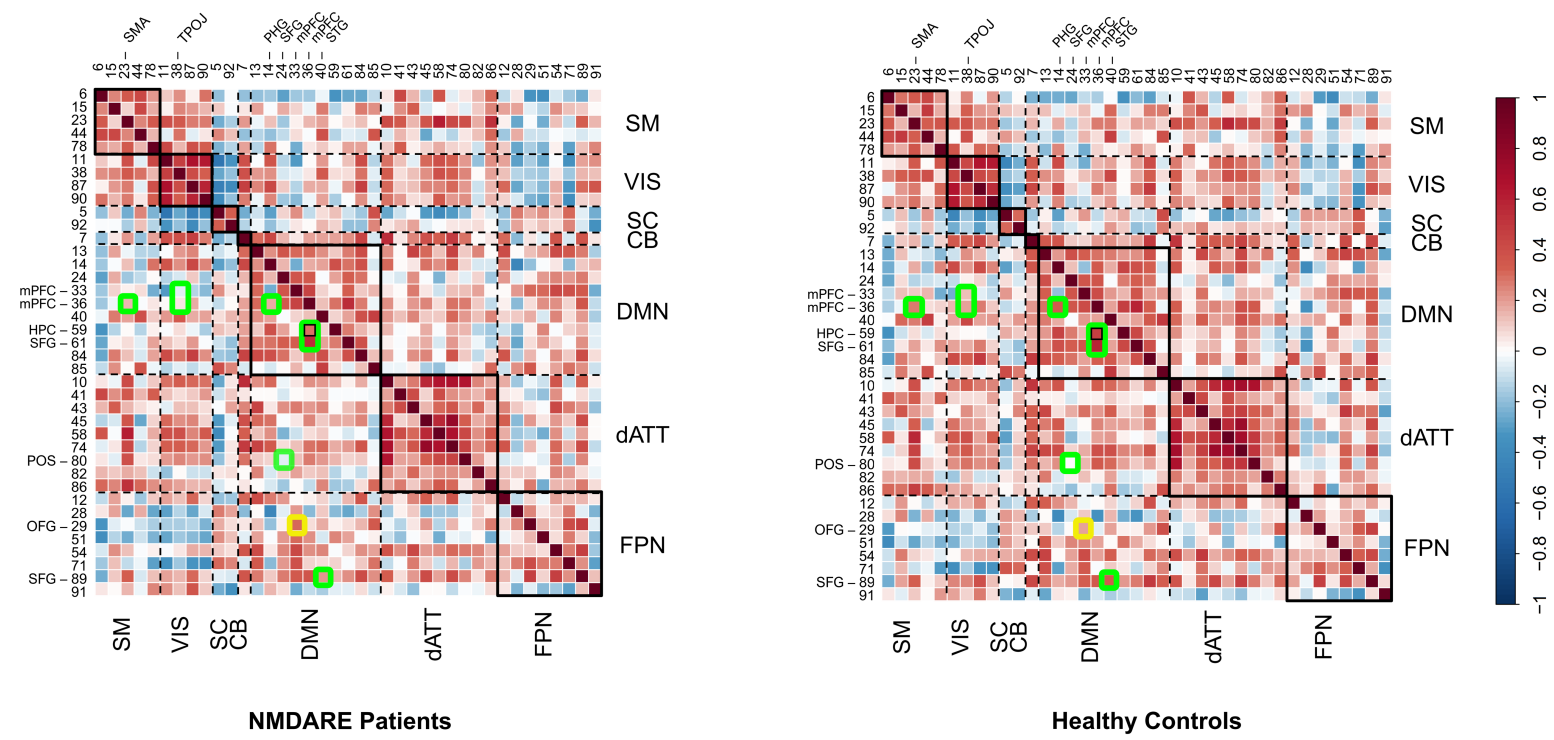

Figure 1: Mean static functional connectivity matrix across brain regions of NMDARE patients (left) and healthy controls (right). Darker red/blue colors indicate higher positive/negative correlation values between component pairs. Green circles mark lower correlation values in NMDARE compared to controls and yellow circles indicate higher correlation values in NMDARE compared to controls. Small black rectangle indicates significant difference of FC between the hippocampus (region 59) and the medial prefrontal cortex (region 36) between patients and controls after FDR-correction ( $p_{F D R}<0.05$ ), while no rectangle indicates differences between groups for puncorr $<0.001$. Highlighted regions are displayed with anatomical labels. A key for the region numbers is provided in Supplementary Table 1. Big diagonal rectangles indicate functional networks, e.g. the sensorimotor network (SM) that is comprised of the regions $6,15,23,44$ and 78 . SM = sensorimotor network; VIS = visual network; SC = subcortical network; $\mathrm{CB}=$ cerebellar network; $\mathrm{DMN}=$ default mode network; $\mathrm{dATT}=$ dorsal attention network; FPN = fronto-parietal control network; HPC = hippocampus; $\mathrm{mPFC}=$ medial prefrontal cortex; PHG = parahippocampal gyrus; OFG = orbito-frontal gyrus; $\mathrm{POS}=$ parieto-occipital gyrus; $\mathrm{SFG}=$ dorsolateral superior frontal gyrus; SMA = supplementary motor area; STG = superior temporal gyrus; TPOJ = temporo-parietal-occipital junction. 
Table 2: Test results of static function network analysis. Table includes component name, network assignment, number (\#), t-value, p-value and effect size (d) of component pairs that are highlighted in Figure 1. $\mathrm{mPFC}=$ medial prefrontal cortex; $\mathrm{PHG}=$ parahippocampal gyrus; $\mathrm{OFG}=$ orbito-frontal gyrus; $\mathrm{POS}=$ parieto-occipital gyrus; $\mathrm{SFG}=$ dorsolateral superior frontal gyrus; $\mathrm{SMA}=$ supplementary motor area; STG $=$ superior temporal gyrus $=$ TPOJ $=$ temporo-parietal-occipital junction. ${ }^{*}$ significant after FDR-correction

\begin{tabular}{ccccccc}
\hline regions & network & component \# & $\boldsymbol{p}_{\text {uncorr }}$ & $\boldsymbol{p}_{\text {FDR }}$ & $\mathbf{T}$ & $\mathbf{d}$ \\
\hline $\mathrm{mPFC}-\mathrm{Hippocampus}$ & $\mathrm{DMN}-\mathrm{DMN}$ & $36-59$ & $<.0001^{*}<.001^{*}$ & 4.36 & .62 \\
\hline $\mathrm{mPFC}-\mathrm{SMA}$ & $\mathrm{DMN}-\mathrm{SM}$ & $36-23$ & .0009 & .151 & 3.30 & .44 \\
\hline $\mathrm{mPFC}-\mathrm{TPOJ}$ & $\mathrm{DMN}-\mathrm{VIS}$ & $33-38$ & .0008 & .151 & 3.26 & .54 \\
\hline $\mathrm{mPFC}-\mathrm{TPOJ}$ & $\mathrm{DMN}-\mathrm{VIS}$ & $36-38$ & .0006 & .151 & 3.40 & .45 \\
\hline $\mathrm{mPFC}-\mathrm{PHG}$ & $\mathrm{DMN}-\mathrm{DMN}$ & $36-14$ & .0002 & .118 & 3.85 & .55 \\
\hline $\mathrm{SFG}-\mathrm{POS}$ & $\mathrm{DMN}-\mathrm{dATT}$ & $24-80$ & .0007 & .151 & 3.29 & .52 \\
\hline $\mathrm{mPFC}-\mathrm{OFG}$ & $\mathrm{DMN}-\mathrm{FPN}$ & $33-29$ & .0004 & .151 & -3.48 & -.37 \\
\hline $\mathrm{mPFC}-\mathrm{SFG}$ & $\mathrm{DMN}-\mathrm{DMN}$ & $36-61$ & .0009 & .151 & 3.27 & .40 \\
\hline $\mathrm{STG}-\mathrm{SFG}$ & $\mathrm{DMN}-\mathrm{FPN}$ & $40-89$ & .0005 & .151 & 3.24 & .49 \\
\hline
\end{tabular}

\section{Dynamic functional network connectivity analysis}

The k-means clustering identified four connectivity states for HC and NMDARE patients. Cluster centroids of these states, i.e. the center of the clusters, are shown in Figure 2 (22). In general, state 1 exhibited the weakest inter- and intra-connectivity of functional domains, followed by state 4 , while between-component connectivity was higher in states 2 and 3 . In contrast to state 1 , state 4 exhibited higher antagonism between the components of most networks.

Dynamic FC alterations varied between NMDARE patients and controls across the distinct connectivity states (Figure 2 and Table 3). As in the static FC group analysis, differences between groups mainly comprised components of the DMN, VIS and FPN, but in a statedependent fashion: patients with NMDARE displayed decreased connectivity between components of the mPFC and the hippocampus in state 1. State 2 showed a connectivity decrease between the MPFC and the angular gyrus as well as the parieto-occipital sulcus in patients. Furthermore, the inferior frontal gyrus exhibited hypoconnectivity to the putamen while being hyperconnected to the primary visual cortex in patients compared to HC. Similarly, state 3 was characterized by decreased connectivity from the inferior frontal gyrus to the putamen. Additionally, connectivity between the TPO-junction and the superior frontal gyrus was reduced in NMDARE patients compared to HC. No significant alterations were observed for state 4 after FDR-adjustment. 
bioRxiv preprint doi: https://doi.org/10 1101/2020 06 12 141945; this version posted June 12 2020. The copyright holder for this preprint (which was not certified by peer review) is the author/funder. All rights reserved. No reuse allowed without permission.
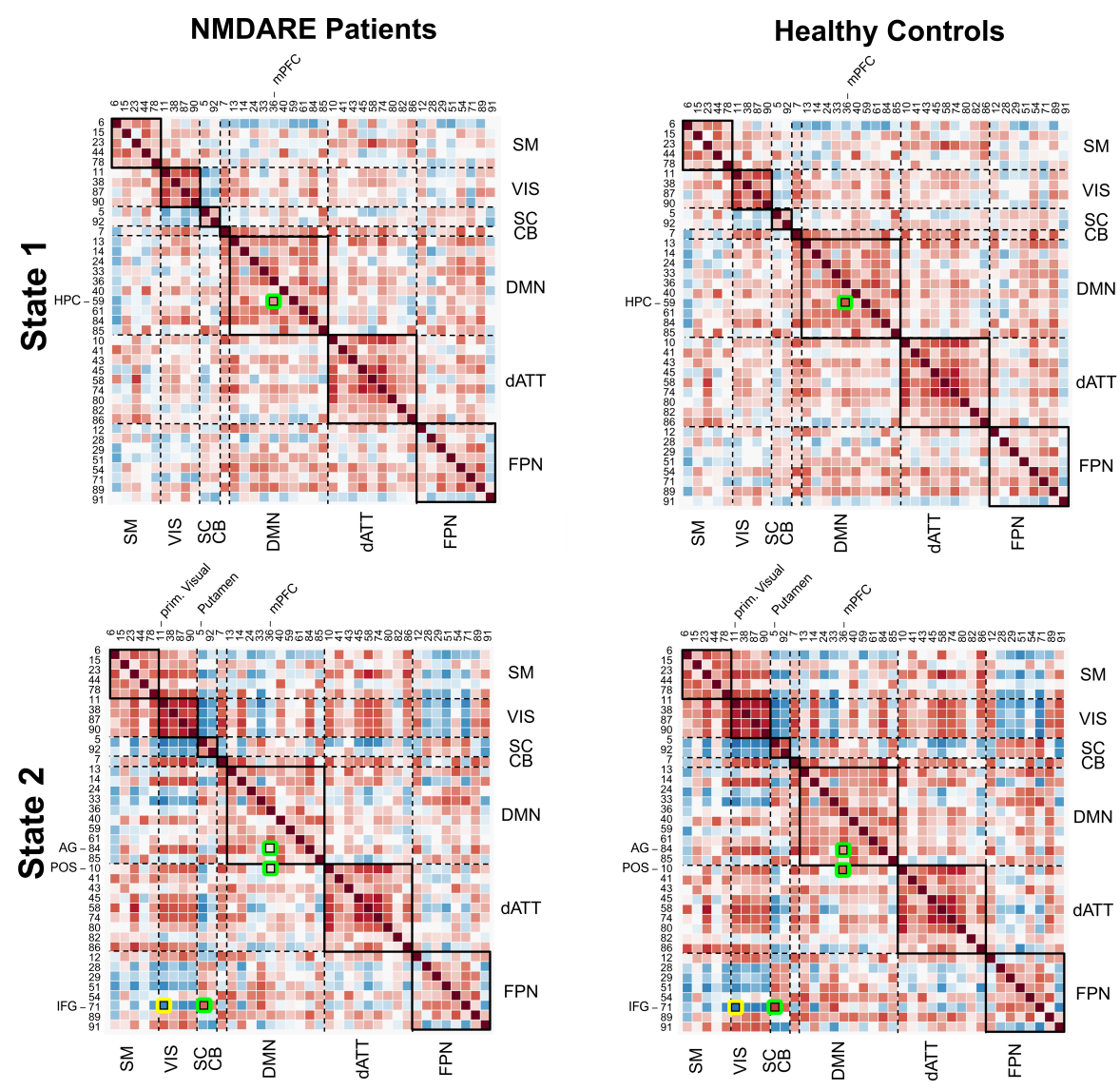

Q
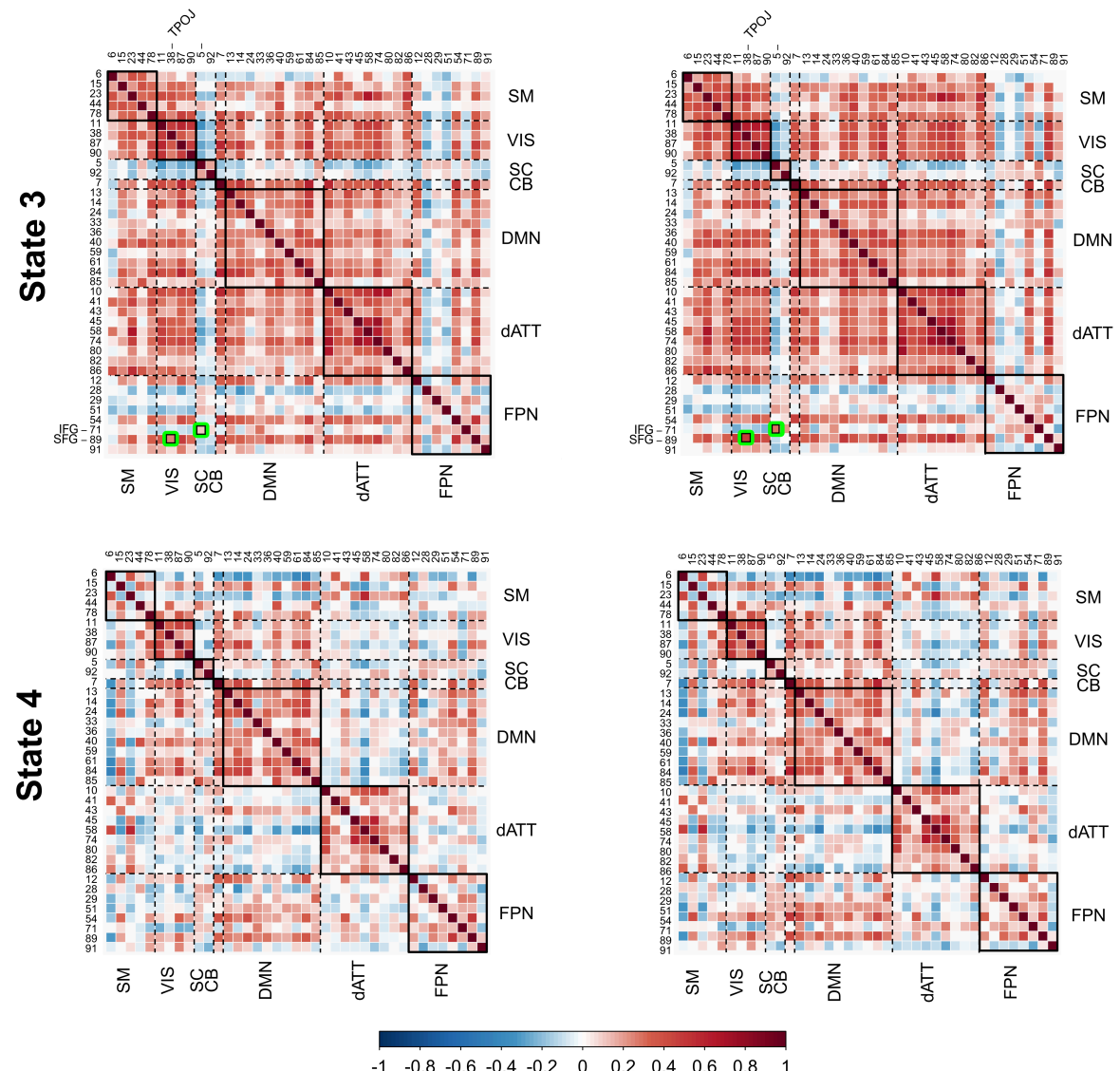

Figure 2: Dynamic functional network connectivity states for NMDARE patients (left) and healthy controls (right). Darker red/blue colors indicate higher positive/negative correlation values between component pairs. Green circles mark lower correlation values in NMDARE compared to controls and 
yellow circles indicate higher correlation values in NMDARE compared to controls. Small black rectangles indicate significant differences of FC between patients and controls after FDR-correction ( $\left.p_{F D R}<0.05\right)$. Highlighted regions are displayed with anatomical labels. A key for the region numbers is provided in Supplementary Table 1. Big diagonal black rectangles indicate functional networks, e.g. the sensorimotor network (SM) that is comprised of the regions $6,15,23,44$ and $78 . \mathrm{SM}=$ sensorimotor network; VIS = visual network; $\mathrm{SC}=$ subcortical network; $\mathrm{CB}=$ cerebellar network; $\mathrm{DMN}=$ default mode network; dATT = dorsal attention network; FPN = fronto-parietal control network; $A G=$ angular gyrus; IFG = inferior frontal gyrus; $\mathrm{mPFC}=$ medial prefrontal cortex; $\mathrm{POS}=$ parieto-occiptal sulcus; prim. visual = primary visual cortex; SFG = superior frontal gyrus; TPOJ- temporo-parietal-occipital junction.

Table 3: Test results of dynamic functional network analysis. Table includes component name, network assignment, number (\#), t-value, p-value and effect size (d) of component pairs that are highlighted in Figure 2. IFG = inferior frontal gyrus; $\mathrm{mPFC}=$ medial prefrontal cortex; $\mathrm{POS}=$ parieto-occiptal sulcus; prim. Visual $=$ primary visual cortex; $\mathrm{SFG}=$ superior frontal gyrus; $\mathrm{TPOJ}=$ temporo-parietal-occipital junction.

\begin{tabular}{|c|c|c|c|c|c|c|}
\hline & Regions & network & $\begin{array}{c}\text { component } \\
\#\end{array}$ & $p_{F D R}$ & $\mathbf{T}$ & d \\
\hline State 1 & mPFC - Hippocampus & DMN - DMN & $36-59$ & .002 & 4.01 & .60 \\
\hline \multirow{4}{*}{ State 2} & prim. Visual - IFG & VIS - FPN & $11-71$ & .016 & -3.80 & -.57 \\
\hline & Putamen - IFG & SC - FPN & $5-71$ & .016 & 4.09 & .56 \\
\hline & mPFC - angular gyrus & DMN - DMN & $36-84$ & .016 & 3.83 & .54 \\
\hline & mPFC - POS & DMN - dATT & $36-10$ & .016 & 4.06 & .79 \\
\hline \multirow{2}{*}{ State 3} & TPOJ - SFG & VIS - FPN & $38-89$ & $<.001$ & 4.33 & .78 \\
\hline & Putamen - IFG & SC - FPN & $5-71$ & .041 & 3.99 & 69 \\
\hline
\end{tabular}

Post-hoc correlation analyses between mRS at the time of scan and the connectivity between the $\mathrm{mPFC}$ and the hippocampus in state 1 revealed no significant relationship $(R=0.18, p=$ 0.237). On an exploratory basis, we obtained the correlation coefficient between all significant component pairs and disease severity (mRS at the time of scan) as well as disease duration (days in hospitalization): In state 2 , higher disease severity was significantly associated with a decrease in FC between mPFC and angular gyrus $(R=-0.40, p=0.013)$, while in state 3 , longer disease duration was significantly related to a decrease in connectivity between TPOJ and SFG $(R=-0.60, p=0.014)$. Post-hoc correlation analyses were not corrected for multiple comparison.

In addition to state-wise connectivity patterns, we assessed group differences in occurrence rate, dwell time and transition frequency. Occurrence rates of dynamic FC states were similar in NMDARE patients and HC: State 1 showed the highest occurrence, followed by states 2 and 4; the lowest occurrence rates were observed for state 3 in both groups. However, statewise between-group proportion tests revealed that state 2 was visited by a higher proportion 
of patients $(N=42,73.68 \%)$ compared to controls $(N=31,50.82 \% ; z=2.34, p=0.019)$, while the proportions were equal for both groups in states 1 (NMDARE: $\mathrm{N}=49,85.96 \%$ vs HC: $\mathrm{N}=$ $55,90.16 \% ; z=.70, p=0.484$ ), 3 (NMDARE: $N=28,49.12 \%$ vs HC: $N=28,45.90 \%$; $z=-$ $.35, p=0.726$ ) and 4 (NMDARE: $N=34,59.65 \%$ vs $\mathrm{HC}: \mathrm{N}=31,50.82 \% ; z=-.96, p=0.337$ ). We also observed differences in the time spent in distinct connectivity states between NMDARE patients and controls. Patients showed significantly lower dwell times in state 1 in comparison to controls $\left(\mathrm{p}_{\mathrm{FDR}}=0.03\right)$. In contrast, patients had time higher dwell times in state 2 compared to controls $\left(p_{F D R}=0.02\right.$; Figure $3 \&$ Table 4 ). Furthermore, NMDARE patients exhibited higher transition frequencies between state 1 and $2\left(p_{F D R}=0.04\right)$ and between state 3 and $4\left(p_{\mathrm{FDR}}<0.01\right.$; Figure 3 and Table 4$)$.

To identify a relationship between disease severity variables (i.e. acute days in hospitalization and mRS score at the time of scan) and dynamic metrics (i.e. dwell time and transition frequency), we conducted a post hoc Pearson's correlation analysis between these variables. We found that increased transition frequency between state 1 and 2 was significantly associated with disease severity at the time of scan $\left(p_{\text {uncorr }}<0.01\right)$, while increased transition frequency between state 3 and 4 was significantly correlated with disease duration (days in hospitalization) in NMDARE patients ( $p_{\text {uncorr }}=0.03$; Table 5 ).
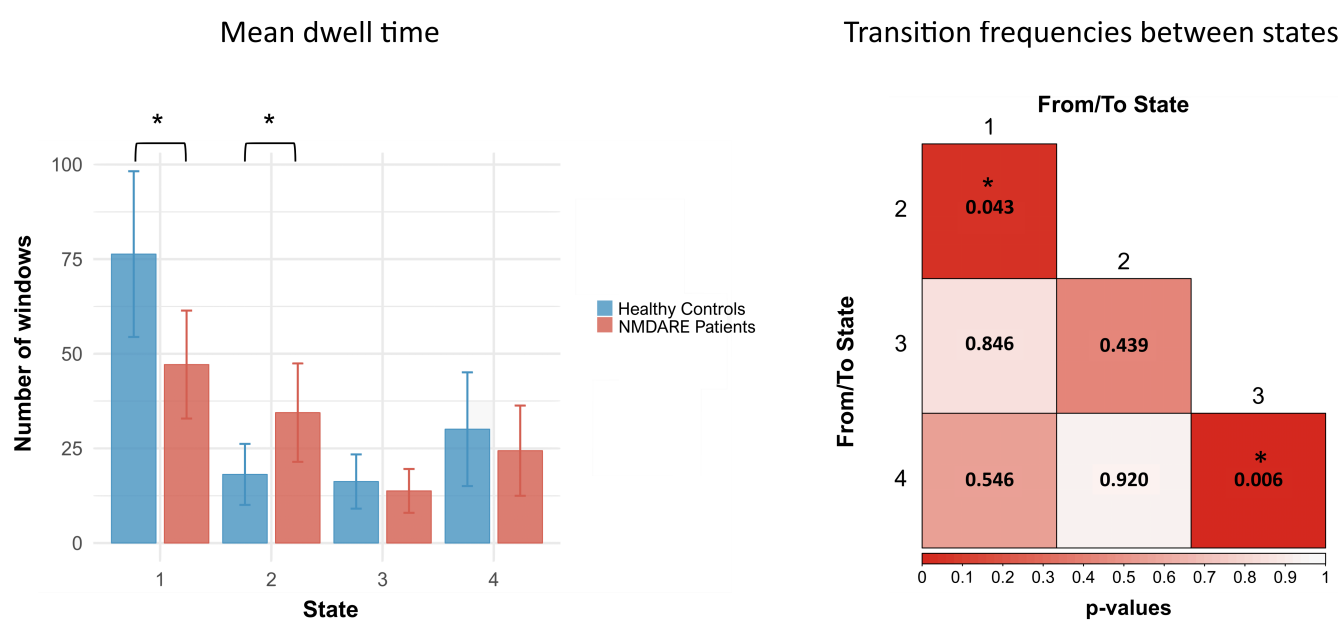

Figure 3: Group differences in average dwell time (in windows) and transition frequencies between states (p-values). For transition frequencies, the direction of transition was ignored. * significant after FDR correction. 
Table 4: Group differences in dwell time (windows) and transition frequencies between states. Group differences were calculated using a two-sided permutation test- p-values and effect sizes (d) are shown. * indicates significant correlations.

\begin{tabular}{cccccc}
\hline & State & $\begin{array}{c}\text { NMDARE Patients } \\
(\text { mean } \pm \text { SD })\end{array}$ & $\begin{array}{c}\text { Healthy Controls } \\
(\text { mean } \pm \text { SD })\end{array}$ & p pDR & $\mathbf{d}$ \\
\hline \multirow{3}{*}{ Dwell time } & 1 & $46.6 \pm 53.8$ & $75.7 \pm 85.6$ & $0.032^{*}$ & 0.42 \\
& 2 & $34.9 \pm 49.9$ & $17.6 \pm 30.6$ & $0.022^{*}$ & -0.43 \\
& 3 & $14.2 \pm 22.8$ & $16.2 \pm 28.0$ & 0.686 & 0.08 \\
Transition frequency & 4 & $24.3 \pm 45.0$ & $30.3 \pm 58.8$ & 0.534 & 0.12 \\
\hline & $1-2$ & $1.3 \pm 1.5$ & $0.8 \pm 1.37$ & $0.043^{*}$ & -0.34 \\
& $1-3$ & $0.8 \pm 1.4$ & $0.8 \pm 1.42$ & 0.846 & 0.03 \\
& $2-4$ & $1.3 \pm 1.9$ & $1.1 \pm 1.95$ & 0.546 & -0.11 \\
& $3-4$ & $0.5 \pm 1.3$ & $0.7 \pm 1.31$ & 0.439 & 0.14 \\
& $1-4$ & $0.3 \pm 0.8$ & $0.3 \pm 0.95$ & 0.920 & 0.01 \\
& & & $0.0 \pm 0.13$ & $0.006^{*}$ & -0.50 \\
\hline
\end{tabular}

Table 5: Correlation of dynamic metrics with disease severity variables. Disease duration $=$ days in hospitalization; $\mathrm{mRS}=$ modified Rankin scale. ${ }^{*}$ significant correlations (uncorrected).

\begin{tabular}{|c|c|c|c|c|}
\hline Metric & mRS at scan ${ }^{\circledR}$ & $\mathbf{p}$ & Disease duration & $\mathbf{p}$ \\
\hline Transition frequency $1-2$ & 0.38 & $0.007^{*}$ & -0.01 & 0.965 \\
\hline Transition frequency 3-4 & -0.04 & 0.757 & 0.37 & $0.033^{*}$ \\
\hline Dwell time in state 1 & -0.09 & 0.535 & -0.12 & 0.504 \\
\hline Dwell time in state 2 & 0.10 & 0.510 & 0.16 & 0.365 \\
\hline
\end{tabular}

\section{Classification analyses}

Binary classification (NMDARE patients vs. $\mathrm{HC}$ ) based on static connectivity features yielded an overall prediction accuracy of $72 \%$, with balanced feature distribution across the networks (see Supplementary Figure 3). When dynamic connectivity features were considered, discriminatory power differed in a state-wise fashion. Prediction performance was lowest for state 1 (overall accuracy of $61.5 \%$ ), intermediate and similar to model performance with static feature input for states 2 (72.6\%) and 4 (70.8\%), and highest for state 3 (78.6\%; see Supplementary Figure 4 for the state-wise confusion matrices). Besides model evaluation outcomes, the feature selection frequencies over individual predictions in the LOOCV scheme also varied across states (Figure 4). While states 1 and 3 yielded balanced selection rates over both across- and within-network connectivity features, states 2 and 4 showed fewer 
discriminatory features, and these were primarily across-network connections (FPN to VIS and DMN for state 2, and DMN to VIS for state 4). Importantly, although some connectivity features were discriminatory across several states (e.g., component pair 12-90 showed high selection frequency for states 1-3), the constellation of predictive features changed dynamically over connectivity states, such that there is no spatial cluster of connectivity features that are always predictive. Furthermore, there was no evident relationship between model performance and the number of features with non-zero selection frequency.

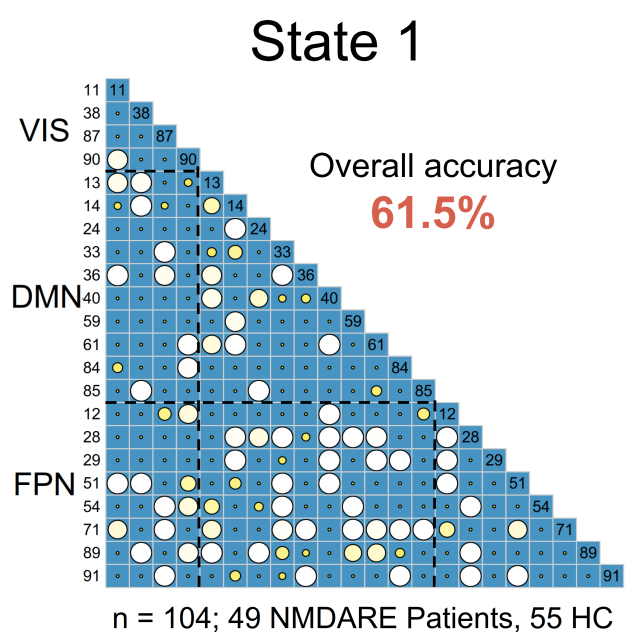

State 3

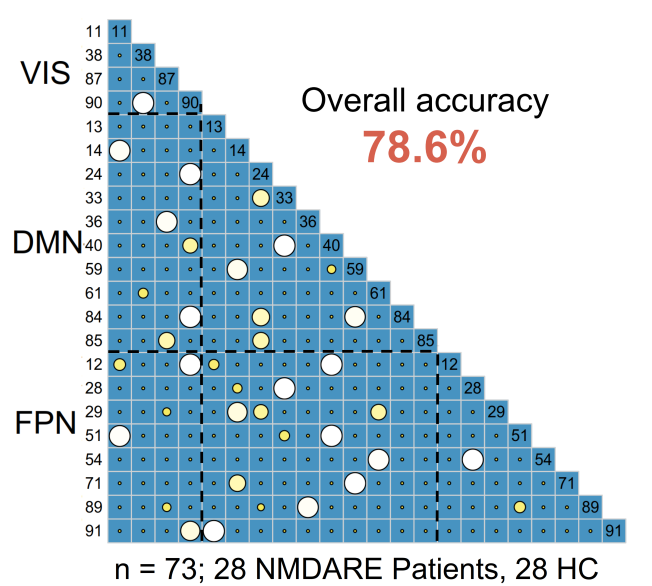

State 2

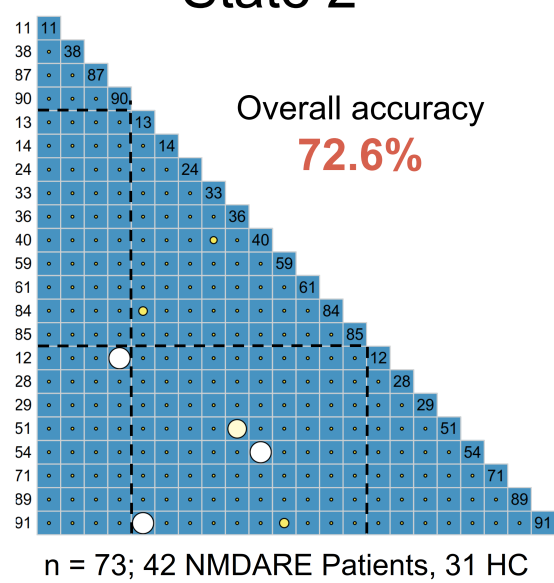

State 4

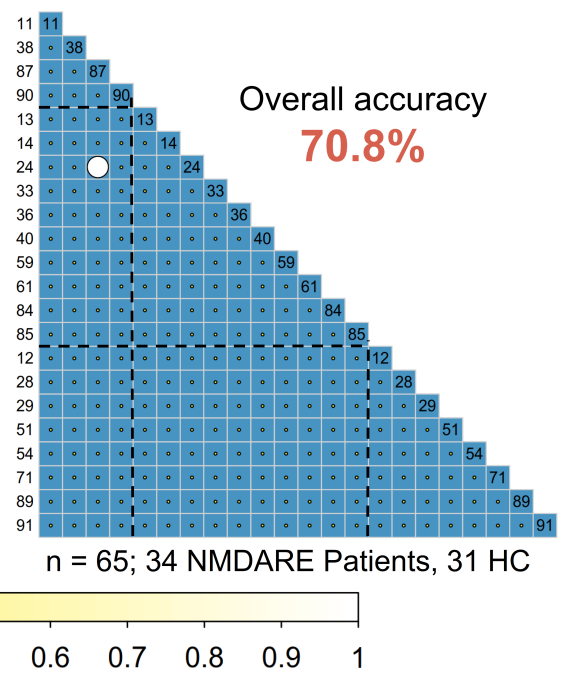

Figure 4: Feature selection matrices for state-wise predictions of group status (NMDARE patients vs healthy controls). The feature selection exceeding a minimum threshold at $10 \%$ of individuals within state predictions are displayed. Bigger and brighter circles indicate a higher selection rate. A key for the region numbers is provided in Supplementary Table 1 . VIS = visual network; DMN = default mode network; dATT = dorsal attention network; FPN = fronto-parietal control network; $\mathrm{HC}=$ healthy controls. 


\section{DISCUSSION}

In this study, we investigated the temporal dynamics of resting-state FC in anti-NMDA receptor encephalitis (NMDARE). Our analyses corroborate earlier findings of static connectivity alterations in NMDARE $(3,8)$ and provide the first characterization of dynamic brain states in this patient population. Our results show state-specific FC disruptions in patients that include a marked reduction in hippocampal-frontal connectivity as well as connectivity decreases within the default mode network and between frontal areas. Along with these connectivity alterations, patients a show a systematic shift in dwell time and increased volatility of state transitions, that were associated with disease severity and duration. Furthermore, classification analysis revealed state-dependent differences in discriminatory power. Taken together, our findings highlight the importance of state-specific characteristics and time-resolved analysis of FC for disease characterization of NMDARE.

We first conducted static functional network analysis, which showed altered connectivity patterns primarily in frontal parts of the DMN as well as decreased connectivity between the mPFC and the hippocampus. This finding is in line with earlier studies that observed reduced FC between the hippocampus and the anterior DMN in patients with $\operatorname{NMDARE}(3,8)$. Histological evidence indicates that the highest density of NMDA receptors is found in the CA1 subregion of the hippocampus and in the prefrontal cortex (31). The converging reports of disrupted hippocampal-frontal connectivity therefore are biologically plausible and point to a disease biomarker and potential treatment target. Furthermore, the hippocampus is functionally connected with the mPFC - a major component of the DMN involved in memory and executive functions (32) - the two cognitive domains that are most frequently impaired in patients with NMDARE $(5,33)$. In accordance with impaired executive function in NMDARE patients (5), we also observed connectivity alterations within the FPN and frontal parts of the DMN (34).

While these findings are thus well in line with previous evidence, they are inherently limited to a static account of connectivity changes. Time-varying FC, in contrast, captures moment-tomoment changes in connectivity reflecting a more physiologically plausible model of brain activity. Therefore, models that study brain activity at the timescale of seconds rather than minutes are necessary to capture the brains' innate dynamics. One line of thought hypothesizes that the temporal variability of FC networks enables a systematic exploration of network configurations, which allows brain regions to dynamically (dis-)engage and modulates changes in cognition and behavior $(35,36)$. Dynamic state analysis $(22)$ as employed in this study represents a powerful tool to describe these dynamics and potential instabilities of this process. 
Dynamic, i.e. state-wise, group comparisons yielded connectivity differences between patients and controls in three out of the four states. Generally, group differences were most pronounced in within- and across-network connectivity of DMN regions, and significant differences were almost exclusively due to a reduction in connectivity strength in NMDARE. Importantly, however, each state showed a characteristic pattern of connectivity alterations highlighting the uniqueness of each individual state. State 1 was marked by a decrease in connectivity strength between the mPFC and the hippocampus. Since state 1 represents the dominant connectivity state, that was visited by the majority of participants and in which they spent most of the time, this reduction may constitute the driving force behind the finding in the static analysis. In contrast, the connectivity reduction within components of the DMN and between frontal FPN areas and visual as well as subcortical components was specific to states 2 and 3 , such that these effects only became apparent in time-resolved analysis, but not in static FC.

Furthermore, our analyses showed a systematic shift in dwell times from this dominant state 1 to the non-dominant state 2 for patients with NMDARE: Patients nearly halved their dwell time in state 1 , while doubling it in state 2 . Along with higher dwell time in state 2, the proportion of patients visiting state 2 was higher compared to HC. Additionally, patients showed an increase in transition frequencies between states 1 and 2 as well as states 3 and 4 , pointing to an increased volatility of network connectivity in NMDARE. The dynamic interplay of brain regions - in the sense of the flexible (dis-)engagement of functional links and state transitions - is critical to efficiently process internal and external stimuli and flexibly adapt behavior. While state transitions are thought to be generally important to explore different brain states in order to facilitate and enhance cognitive flexibility $(35,36)$, overly unstable transition dynamics may be linked to deficiencies in the integration $(21,37)$ and stable representation of information (38). That is, the imbalance of stability and volatility may lead to impaired memory (39), perception (17) or executive functions (21).

While our findings thus support the role of the hippocampus, the anterior DMN, and frontal areas as potential biomarker targets in NMDARE, group-level analyses are not suited to estimate the discriminatory power of connectivity alterations or their value to predict disease severity (40). To this end, we applied classification analyses based on logistic regression models to these data, which distinguished patients from controls with up to $78.6 \%$ accuracy (as compared to $72 \%$ accuracy in the static model). Prediction performance and the set of selected network features were variable across the different connectivity states, indicating that discriminatory network constellations differ from state to state. This is in accordance with recent studies showing that incorporating temporal information can be useful in classification approaches (41) and further emphasizes the benefit of state-specific connectivity alterations over routine structural MRI as clinical and/or prognostic markers. 
Furthermore, disease severity at the time of scanning was negatively associated with indices of both static and dynamic FC, primarily involving the mPFC. Similarly, dynamic metrics also showed significant correlations with disease severity variables: increased transition frequency between states 1 and 2 correlated with disease severity, while the increase of transition frequency between states 3 and 4 correlated with disease duration. These findings raise the intriguing possibility that disrupted resting-state dynamics could carry prognostic information in NMDARE.

In this context, our findings show an interesting convergence with recent studies in schizophrenia reporting that the fraction of time patients spent in a state similarly shifted from one state to another $(13,29)$, while they also exhibit higher overall transition frequencies $(42)$. Since there is considerable overlap with schizophrenia in the psychiatric manifestations of NMDARE (6) and NMDA receptor dysfunction is discussed as a pathophysiological basis for cognitive and positive symptoms in schizophrenia (43) our findings raise the question if the transdiagnostic psychopathological profile in NMDARE $(44,45)$ could be paralleled by a common set of network alterations, such as more volatile transition dynamics.

Finally, some limitations of the present study deserve mentioning. First, window-based approaches require the specification of windowing parameters, and the optimal choices in this regard are an active area of research and debate $(15,46)$. Here, we followed the guidelines of (47) in choosing a window size of 64.5 seconds. Furthermore, a given window size may only capture a part of the dynamic nature of the human brain, as networks may reconfigure over different time scales even within the possible temporal spectrum of MRI signals (48). Moreover, for classification analyses it is generally sensible to include larger amount of data (40). While our study is based on a large study population, the sample sizes per state varied because not all participants visited all states.

\section{Conclusion}

Dynamic FC analyses revealed state-specific alterations of network connectivity in NMDARE. Besides a marked reduction in hippocampal-frontal connectivity, this time-resolved approach yielded additional connectivity decreases within the DMN and between frontal areas and subcortical as well as visual regions, which remain undetected in static FC analysis. Additionally, patients showed a systematic shift in dwell time from the dominant state to a nondominant state as well as an increased volatility of state transitions, and these dynamic metrics were associated with disease severity and duration. In sum, this study emphasizes the importance of including temporal dynamics in the characterization of NMDARE and highlights the complex interplay of inter- and intrastate network alterations. 


\section{REFERENCES}

1. Dalmau J, Lancaster E, Martinez-Hernandez E, Rosenfeld MR, Balice-Gordon R. Clinical experience and laboratory investigations in patients with anti-NMDAR encephalitis. Lancet Neurol 2011;10:63-74.

2. KAYSER MS, DALMAU J. Anti-NMDA receptor encephalitis, autoimmunity, and psychosis. Schizophr Res 2016;176:36-40.

3. FINKE C, KOPP UA, SCHEEL M et al. Functional and structural brain changes in anti-Nmethyl-D-aspartate receptor encephalitis: Finke et al: MRI in Anti-NMDAR Encephalitis. Ann Neurol 2013;:n/a-n/a.

4. LISMAN JE, FELLOUS JM, WANG XJ. A role for NMDA-receptor channels in working memory. Nat Neurosci 1998;1:273-275.

5. Finke C, Kopp UA, Prüss H, Dalmau J, WANDinger K-P, Ploner CJ. Cognitive deficits following anti-NMDA receptor encephalitis. J Neurol Neurosurg Psychiatry 2012;83:195-198.

6. DALMAU J, Graus F. Antibody-Mediated Encephalitis. N Engl J Med 2018;378:840851.

7. Heine J, Prüss H, Bartsch T, Ploner CJ, PAUl F, Finke C. Imaging of autoimmune encephalitis - Relevance for clinical practice and hippocampal function. Neuroscience 2015;309:68-83.

8. PeER M, PRÜSs H, Ben-DAYAN I, PAUl F, ARZY S, FinKe C. Functional connectivity of large-scale brain networks in patients with anti-NMDA receptor encephalitis: an observational study. Lancet Psychiatry 2017;4:768-774.

9. BISWAL B, ZERRIN YETKIN F, HAUGHTON VM, HYDE JS. Functional connectivity in the motor cortex of resting human brain using echo-planar mri. Magn Reson Med 1995;34:537-541.

10. Calmoun VD, Miller R, Pearlson G, Adali T. The Chronnectome: Time-Varying Connectivity Networks as the Next Frontier in fMRI Data Discovery. Neuron 2014;84:262274.

11. HutChiSON RM, WOMELSDORF T, AlLEN EA et al. Dynamic functional connectivity: Promise, issues, and interpretations. Neurolmage 2013;80:360-378.

12. ChANG C, GLOVER GH. Time-frequency dynamics of resting-state brain connectivity measured with fMRI. Neurolmage 2010;50:81-98.

13. Damaraju E, Allen EA, Belger A et al. Dynamic functional connectivity analysis reveals transient states of dysconnectivity in schizophrenia. Neurolmage Clin 2014;5:298308.

14. ZALESKY A, Fornito A, COCCHI L, GOLLO LL, BREAKSPEAR M. Time-resolved restingstate brain networks. Proc Natl Acad Sci U S A 2014;111:10341-10346.

15. LURIE DJ, KESSLER D, BASSETT DS et al. Questions and controversies in the study of time-varying functional connectivity in resting fMRI. Netw Neurosci 2020;4:30-69. 
16. GONZALEZ-CASTILLO J, HOY CW, HANDWERKER DA et al. Tracking ongoing cognition in individuals using brief, whole-brain functional connectivity patterns. Proc Natl Acad Sci 2015;112:8762-8767.

17. Sadaghiani S, Poline J-B, KLeinschmidt A, D’Esposito M. Ongoing dynamics in large-scale functional connectivity predict perception. Proc Natl Acad Sci 2015;112:84638468.

18. MenNigen E, MiLleR RL, RASHid B et al. Reduced higher-dimensional resting state fMRI dynamism in clinical high-risk individuals for schizophrenia identified by meta-state analysis. Schizophr Res 2018;201:217-223.

19. BRAUN U, SCHÄFER A, BASSETT DS et al. Dynamic brain network reconfiguration as a potential schizophrenia genetic risk mechanism modulated by NMDA receptor function.

Proc Natl Acad Sci 2016;113:12568-12573.

20. EIJLERS AJC, WINK AM, MEIJER KA, DOUW L, GEURTS JJG, SCHOONHEIM MM. Reduced Network Dynamics on Functional MRI Signals Cognitive Impairment in Multiple Sclerosis. Radiology 2019;292:449-457.

21. BRAUN U, SCHÄFER A, WALTER H et al. Dynamic reconfiguration of frontal brain networks during executive cognition in humans. Proc Natl Acad Sci 2015;112:1167811683.

22. Allen EA, damaraju E, Plis SM, Erhardt EB, Eichele t, Calhoun VD. Tracking whole-brain connectivity dynamics in the resting state. Cereb Cortex N Y N 1991 2014;24:663-676.

23. CALHOUN VD, Adali T, PEARLson GD, PEKAR JJ. A method for making group inferences from functional MRI data using independent component analysis. Hum Brain Mapp 2001;14:140-151.

24. HIMBERG J, HYVARINEN A. Icasso: software for investigating the reliability of ICA estimates by clustering and visualization. In: 2003 IEEE XIII Workshop on Neural Networks for Signal Processing (IEEE Cat. No.03TH8718). Toulouse, France: IEEE, 2003, 259-268.

25. DU Y, FAN Y. Group information guided ICA for fMRI data analysis. Neurolmage 2013;69:157-197.

26. THOMAS YEO BT, KRIENEN FM, SEPULCRE J et al. The organization of the human cerebral cortex estimated by intrinsic functional connectivity. J Neurophysiol 2011;106:1125-1165.

27. BartTfELd P, UhRIg L, SitT JD, Sigman M, JaRRAYA B, Dehaene S. Signature of consciousness in the dynamics of resting-state brain activity. Proc Natl Acad Sci 2015;112:887-892.

28. BenJAMINI Y, HOCHBERG Y. Controlling the False Discovery Rate: A Practical and Powerful Approach to Multiple Testing. J R Stat Soc Ser B Methodol 1995;57:289-300.

29. DU Y, PEARLSON GD, YU Q et al. Interaction among subsystems within default mode network diminished in schizophrenia patients: A dynamic connectivity approach. Schizophr Res 2016;170:55-65. 
30. GLEREAN E, PAN RK, SALMI J et al. Reorganization of functionally connected brain subnetworks in high-functioning autism: Reorganization of Subnetworks in Autism. Hum Brain Mapp 2016;37:1066-1079.

31. MONAGHAN D, COTMAN C. Distribution of N-methyl-D-aspartate-sensitive L[3H]glutamate-binding sites in rat brain. J Neurosci 1985;5:2909-2919.

32. MILLER EK, COHEN JD. An Integrative Theory of Prefrontal Cortex Function. Annu Rev Neurosci 2001;24:167-202.

33. MCKEON GL, ROBINSON GA, RYAN AE et al. Cognitive outcomes following anti-Nmethyl-D-aspartate receptor encephalitis: A systematic review. J Clin Exp Neuropsychol 2018;40:234-252.

34. Cole MW, Repovš G, ANTICEVIC A. The Frontoparietal Control System: A Central Role in Mental Health. The Neuroscientist 2014;20:652-664.

35. DECO G, JIRSA VK, MCINTOSH AR. Emerging concepts for the dynamical organization of resting-state activity in the brain. Nat Rev Neurosci 2011;12:43-56.

36. LI J, ZHANG D, LIANG A et al. High transition frequencies of dynamic functional connectivity states in the creative brain. Sci Rep 2017;7:46072.

37. ZHANG J, CHENG W, LIU Z et al. Neural, electrophysiological and anatomical basis of brain-network variability and its characteristic changes in mental disorders. Brain 2016;139:2307-2321.

38. LI L, LU B, YAN C-G. Stability of dynamic functional architecture differs between brain networks and states. Neurolmage 2019;:116230.

39. KUCYI A, TAmbini A, SAdAghiani S, KeILHOLZ S, COHEN JR. Spontaneous cognitive processes and the behavioral validation of time-varying brain connectivity. Netw Neurosci 2018;2:397-417.

40. ARBABSHIRANI MR, PLIS S, SUI J, CALHOUN VD. Single subject prediction of brain disorders in neuroimaging: Promises and pitfalls. Neurolmage 2017;145:137-165.

41. Vergara VM, Mayer AR, Kiehl KA, Calhoun VD. Dynamic functional network connectivity discriminates mild traumatic brain injury through machine learning. Neurolmage Clin 2018;19:30-37.

42. Rashid B, Damaraju E, Pearlson GD, Calhoun VD. Dynamic connectivity states estimated from resting fMRI Identify differences among Schizophrenia, bipolar disorder, and healthy control subjects. Front Hum Neurosci 2014;8. doi:10.3389/fnhum.2014.00897

43. BALU DT. The NMDA Receptor and Schizophrenia. In: Advances in Pharmacology. Elsevier, 2016, 351-382.

44. FINKE C. A transdiagnostic pattern of psychiatric symptoms in autoimmune encephalitis. Lancet Psychiatry 2019;6:191-193.

45. AL-DIWANI A, HANDEL A, TOWNSEND L et al. The psychopathology of NMDARantibody encephalitis in adults: a systematic review and phenotypic analysis of individual patient data. Lancet Psychiatry 2019;6:235-246. 
46. HINDRIKS R, ADHIKARI MH, MURAYAMA Y et al. Can sliding-window correlations reveal dynamic functional connectivity in resting-state fMRI? Neurolmage 2016;127:242-256.

47. LEONARDI N, VAN DE VILLE D. On spurious and real fluctuations of dynamic functional connectivity during rest. Neurolmage 2015;104:430-436.

48. CABRAL J, KRINGELBACH ML, DECO G. Functional connectivity dynamically evolves on multiple time-scales over a static structural connectome: Models and mechanisms. Neurolmage 2017;160:84-96. 